\title{
Country-Level Heavy Episodic Drinking and Individual-Level Experiences of Harm from Others' Drinking-Related Aggression in 19 European Countries
}

\author{
Erica Sundin ${ }^{a, b}$ Jonas Landberg ${ }^{a, c}$ Maria Rosaria Galanti ${ }^{d, e}$ Robin Room ${ }^{c, f}$ \\ Mats Ramstedt ${ }^{\mathrm{a}, \mathrm{b}}$ \\ ${ }^{a}$ Department of Clinical Neuroscience, Karolinska Institutet, Stockholm, Sweden; ${ }^{\text {TT }}$ The Swedish Council for \\ Information on Alcohol and Other Drugs, Stockholm, Sweden; 'Centre for Social Research on Alcohol and Drugs, \\ Department of Public Health Sciences, Stockholm University, Stockholm, Sweden; dDepartment of Global Public \\ Health, Karolinska Institutet, Stockholm, Sweden; ${ }^{\mathrm{e} C e n t r e ~ f o r ~ E p i d e m i o l o g y ~ a n d ~ C o m m u n i t y ~ H e a l t h, ~ S t o c k h o l m ~}$ \\ County Council, Stockholm, Sweden; 'Centre for Alcohol Policy Research, La Trobe University, Melbourne, VIC, \\ Australia
}

\section{Keywords}

Alcohol · Harm to others - Aggression · Heavy episodic drinking $\cdot$ Cross-country

\begin{abstract}
Introduction: There is limited knowledge about how individual experiences of harm from others' drinking are influenced by heavy episodic drinking (HED) at the country level. The present study aimed to assess (1) the association between the country-level prevalence of HED and the risk of experiencing harm from others' drinking-related aggression and (2) if HED at the country level modifies the association between consumption of alcohol per capita (APC) and such harm. Methods: Outcome data from 32,576 participants from 19 European countries stem from the RARHA SEAS study. Self-reported harm from others' drinking included having been verbally abused, harmed physically, or having serious arguments. Data on country-level drinking patterns were derived from the World Health Organization. Associations between country-level prevalence of monthly HED and experiences of aggression (at least 1 of 3 studied harms) were derived through multilevel models - adjusted for coun-
\end{abstract}

karger@karger.com www.karger.com/ear

Karger $\stackrel{\text { ' }}{5}$

GOPEN ACCESS
C 2021 The Author(s).

Published by S. Karger AG, Basel

This article is licensed under the Creative Commons Attribution 4.0 International License (CC BY) (http://www.karger.com/Services/ OpenAccessLicense). Usage, derivative works and distribution are permitted provided that proper credit is given to the author and the original publisher. try-level age structure and by including the respondent's own HED patterns as a mediator. Results: A 1\% increase in the prevalence of monthly HED was associated with $5 \%$ higher odds (odds ratio [OR] 1.05) of experiencing others' alcohol-related aggression among men, and 6\% (OR 1.06) among women. The results suggest that the association between APC and harm was stronger in countries with high prevalences of HED, but the modifying effect could not be confirmed. Discussion/Conclusion: Harm from others' drinking-related aggression depends not only on individual factors but is also influenced by the drinking patterns of the population. However, the country-level prevalence of HED only explains a small part of the variance of this type of harm.

(c) 2021 The Author(s).

Published by S. Karger AG, Basel

\section{Introduction}

There has been a renewed interest in research on alcohol's harm to others (AHTO) among researchers and policymakers during the last decade [1]. Studies in this area apply the perspective of the person experiencing harm 
from another's drinking rather than that of the drinker when addressing the epidemiology of alcohol-related harm. A wide range of harms are examined, commonly using population surveys, with alcohol-related aggression and violence receiving substantial attention [2]. However, previous studies have mainly focused on risk factors for exposure to AHTO at the individual level, failing to take population-level factors into account. This is a limitation since an individual's risk of experiencing harm from others' alcohol-related violence is imbedded in various contextual factors, ranging from the individual's social circles to cultural patterns of drinking at the societal level [3]. In order to tailor effective interventions and policies to reduce experiences of others' alcohol-related aggression, a better understanding of how such societal factors are related to the risk of AHTO is warranted. By using data from 19 European countries, the present study sought to shed light on the importance of heavy episodic drinking (HED) patterns in the population for an individual's risk of being exposed to other people's alcoholrelated aggression. A substantial body of literature describes cultural differences in drinking in Europe (see e.g., $[4,5])$; in the northern and eastern parts, heavy drinking on weekends is more culturally accepted. This is in contrast to the southern parts, where drinking is described as more integrated into everyday life through meals.

It is well established that alcohol consumption, and HED (also referred to as binge drinking or risky single occasion drinking), is involved in various forms of aggressive and violent behaviours [6]. In fact, alcohol affects people's behaviour and judgement in a way that increases the risk of aggression and violence [7] in a dose-related fashion $[8,9]$. The underlying mechanisms behind this association are complex and dependent on both individual and environmental factors [7, 9]. However, a central feature is that alcohol intoxication leads to a loss of control of feelings of anger that are suppressed in a sober state [8]. In addition, studies suggest that HED also increases the risk of being a victim of violence related to other people's drinking [7]. This may be explained by impaired coordination and judgement of risks when under the influence of alcohol, reducing the ability to avoid situations where violence may arise.

Previous ecologic studies have also shown a stronger association between population-wide drinking and indicators of violence in countries where the prevalence of HED is higher. For instance, using time series analyses on aggregate-level data for 1950-1995, Rossow [10] found that the association between alcohol sales and homicide was stronger in northern European countries, where

Heavy Episodic Drinking and Harm from

Others' Drinking-Related Aggression
HED accounted for a higher proportion of drinking occasions than in southern European countries. Bye [11] and Ramstedt [12] reported similar findings with strong associations between alcohol sales and homicide in Eastern Europe and Australia, respectively, where HED also constitutes a high proportion of drinking occasions.

Studies that directly assess the association between HED at the country level and individual level of risk of being a victim of violence are rare. One study based on both national and individual-level data on drinking patterns among adults from 18 countries showed that the drinking patterns at both levels were positively associated with alcohol-related consequences [13]. The authors also found that the association between volume of drinking at the individual level and alcohol-related consequences differed depending on the country's level of detrimental drinking patterns. However, this study did not specifically distinguish the risk of being a victim of violence, as it included a combined measure of injuries and fights. Moreover, it did not focus solely on HED but used a broader measure of drinking patterns. Other cross-national studies at the individual level have demonstrated that violence is more frequent in countries with a high prevalence of HED. However, these studies were limited to adolescents and violence was described from the perspective of the drinker [14].

The association between HED at the country level and the individual risk of being exposed to alcohol-related violence is thus poorly examined and is only supported by indirect findings focussing on volume of drinking. Although earlier findings suggest that HED modifies the association between volume of drinking and indicators of violence, this has, to our knowledge, not yet been empirically tested.

Against this background, using multilevel modelling, we will analyse the link between the country-level drinking pattern and self-reported experiences of harm from others' drinking-related aggression across 19 European countries. More specifically, the aim of this study was to investigate whether the country-level prevalence of HED as an ecologic exposure is related to the individual-level risk of experiencing harm from others' drinking-related aggression. To build on previous research, a secondary aim was to assess if HED modifies the association between volume of drinking, in terms of consumption of alcohol per capita (APC), and self-reported experiences of others' alcohol-related aggression, which has only been indirectly assessed in previous research. Based on the previous presented research on the relationship between consuming larger amounts of alcohol on a drinking occa- 


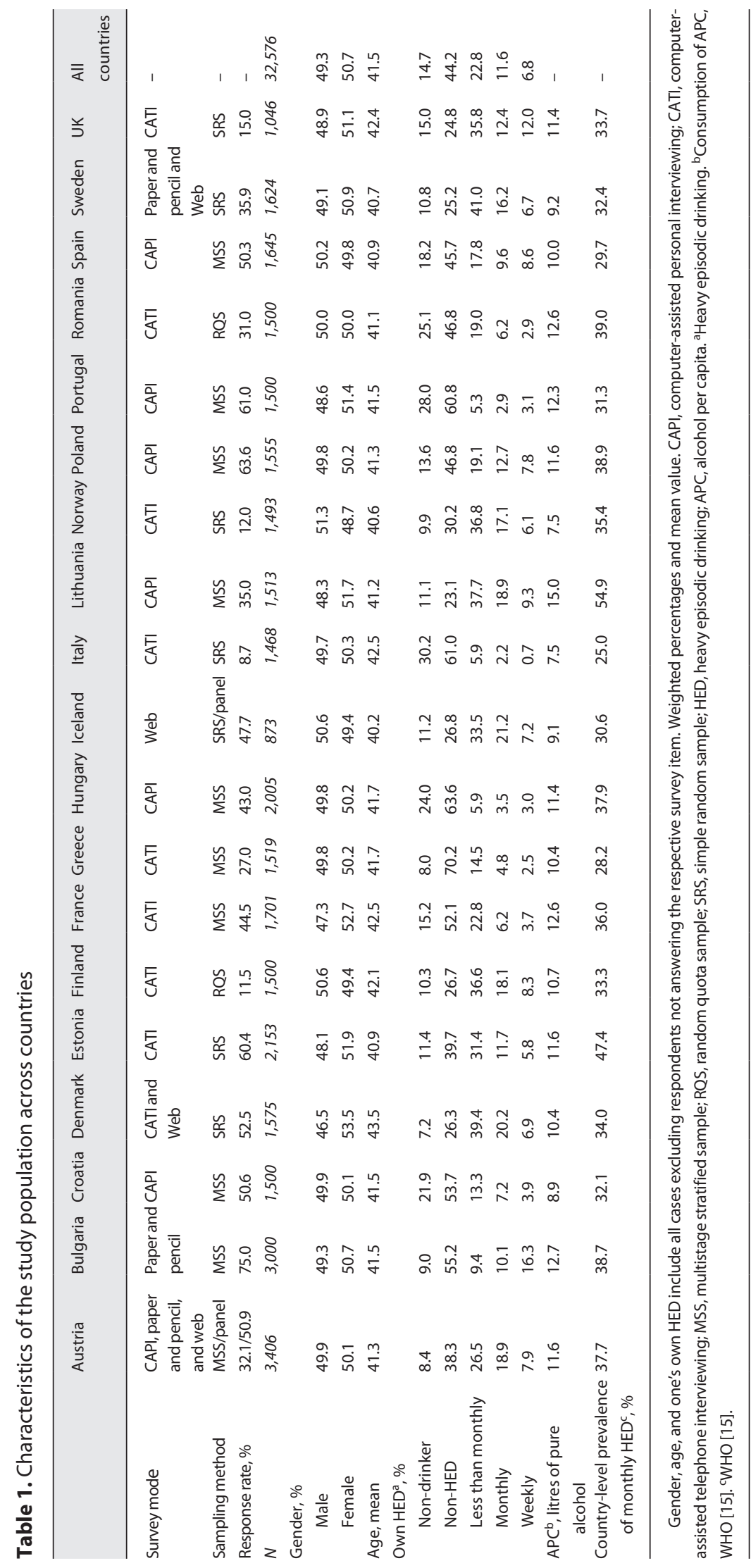


sion and the increased risk of violence, as well as experiences of violence at low levels of alcohol consumption in countries with high proportions of HED, 2 hypotheses were developed: (a) the individual risk of experiencing others' alcohol-related aggressive behaviour is higher in countries where HED is more common, and (b) HED modifies the association between the country-level volume of drinking and experiences of others' alcohol-related aggressive behaviour at the individual level, reflected by a stronger association in countries with higher prevalence of HED.

\section{Materials and Methods}

Cross-sectional data stem from the Joint Action on Reducing Alcohol-Related Harm Standardized European Alcohol Survey (RARHA SEAS), a study conducted in 19 European countries [5]. Self-reported information was collected in 2015 through surveys with random samples from the general population. Most national samples included people aged between 18 and 64, whereas in some countries, individuals aged up to 65 were included.

The sampling method was not standardized due to differences in modes of data collection across countries and the sampling frame was country-specific aiming at random sampling techniques. For a more comprehensive description of these procedures in each country, see the RARHA SEAS report [5]. In general, multistage random sample, random simple sample, and random quota sampling techniques were used (Table 1). In regard to the telephone interviews, registered landlines and/or mobile phone numbers were included in the sampling frame. Moreover, registered administrative units and household addresses were used as sampling frames for the personal interviews. The selection of households varied and included, for example, random route procedures and every second house selection. Random and quota selection as well as the last birthday method are examples of target person selections in the telephone and personal interviews.

The different modes of data collection used were (Table 1): computer-assisted telephone interviewing (8 countries); computer-assisted personal interviewing (6 countries); web questionnaire ( 1 country), paper and pencil (1 country), and mixed modes ( 3 countries). Minimum sample size was set to 1,500 , although in 2 countries samples were smaller. A total of 32,576 individuals participated in the study. A standard questionnaire was translated into national languages and used in all countries. The response rates varied between 8.7 (Italy) and 75\% (Bulgaria) (Table 1).

\section{Outcome}

The questions regarding experiences of harm from others' drinking-related aggression were introduced by a statement emphasizing that the questions concerned "various problems that can occur because of someone else's drinking" and each question started with the phrasing "Because of someone else's drinking...". Respondents were asked whether or not they had experienced the following 3 occurrences during the preceding 12 months: "...have you been verbally abused, for example, called names or otherwise insulted?"/; “...have you been involved in a serious argument?"/; "...have you been harmed physically?". Respondents answering affirmatively to at least one of these items were compared to respondents reporting none of the items.

\section{Exposures and Effect Modifier}

The main exposure measure of the country-level prevalence of monthly HED was derived from an independent source, the World Health Organization (WHO) [15], with data related to 2016. HED was defined as the proportions of the population 15 years and older who consumed $60 \mathrm{~g}$ or more of pure alcohol on at least $1 \mathrm{occa}-$ sion in the past 30 days. The estimates of HED were adjusted for several country-level factors. Additionally, the country-level prevalence of monthly HED was also used as an effect modifier in regard to assessing the secondary aim. In these analyses, a second exposure was used; the countries' total volume of drinking, that is, the consumption of APC in litres of pure alcohol (15+ years population). APC was also available from the WHO [15] and was based on a 3-year average from 2015 to 2017.

\section{Country-Level Variables}

Different levels of self-reported harm across countries may partly reflect different age structures of the countries [16, 17]; therefore, the analyses were adjusted for the country-level mean age according to the RARHA SEAS data.

\section{Individual-Level Variables}

As individual-level drinking habits are important factors for experiencing harm from others' drinking [2], own HED was taken into account as a potential mediator. In RARHA SEAS, own HED patterns were assessed through a question in which the threshold of consumption of pure alcohol on 1 occasion was set to $60 \mathrm{~g}$ or more for men and $40 \mathrm{~g}$ or more for women. The frequency of such episodes of HED during the last 12 months was categorized into: non-drinkers; non-HED; less than monthly; monthly (1-3 times a month); and weekly (1 time or more a week).

\section{Statistical Analyses}

Two-level random effect multilevel mixed-effect binary regression models were used to explore the association between the country-level prevalence of monthly HED and individual-level prediction of experiencing harm from others' drinking-related aggression. Models were fitted both without any adjustment (HED model 1) and after adjustment for country-level age structure and including one's own HED patterns as a mediator (HED model 2). For each model, intraclass correlation was also calculated to assess how much of the total variance of experiencing harm could be accounted for by country-level HED.

In order to assess if country levels of HED modify the association between APC and experiencing harm from others' drinkingrelated aggression, we included an interaction between APC and the country-level prevalence of HED in an additional model (APC and HED model 2) adjusted for country-level age structure and including one's own HED patterns as a mediator. As evaluating the interaction term also requires that the association between APC and others' alcohol-related aggression is first established, we also included 2 models of the association between APC and experiences of aggression related to others' drinking: unadjusted (APC model 1) and adjusted for age structure and including the mediator 


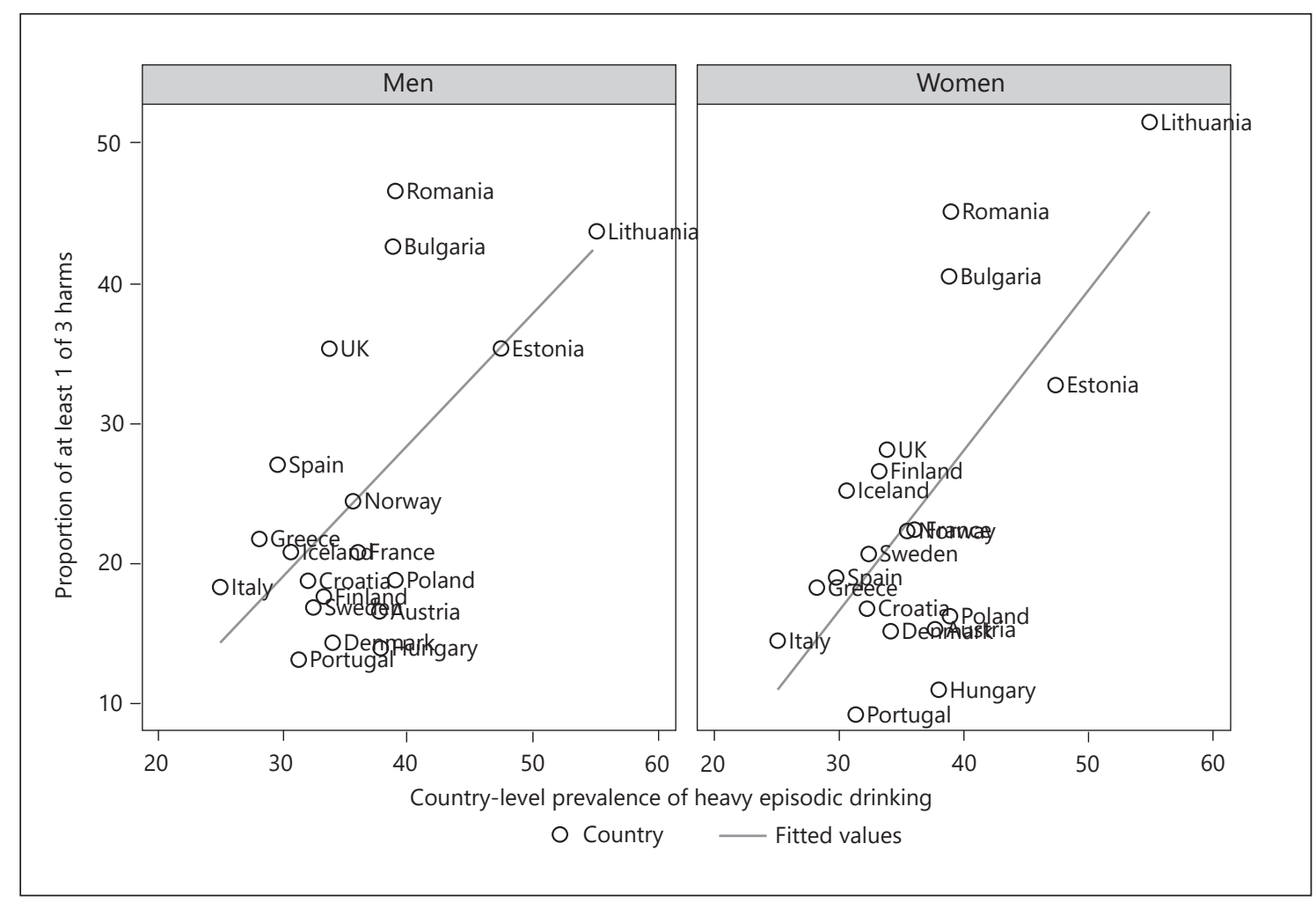

Fig. 1. Prevalence (\%) of experiences of others' alcohol-related aggression during the last year and country-level prevalence of HED. HED, heavy episodic drinking.

of one's own HED patterns (APC model 2). Additionally, the 2 first-order predictors of APC and country-level HED were also first evaluated in the same model without the interaction term (APC and HED model 1).

The predictions of the model including the interaction term were plotted in a graph. The commands for margins and marginsplot in Stata [18] were used to calculate the predicted probability of experiences of others' alcohol-related aggression for APC for every 5 values between 25 and 55 of country-level prevalence of HED.

To avoid collinearity between the interaction term and the first-order predictors (HED and APC), we followed a procedure called residual centring suggested by Lance [19]. Firstly, using ordinary least square regression, the interaction term was regressed on the first-ordered variables, in this case HED and APC. Secondly, the residuals of this regression were used to represent the interaction term in the model (APC and HED model 2). The variance of the interaction term obtained by this regression is independent of the variance of the first-order predictor variable.

Given the large gender differences in involvement in violence, all models were estimated for men and women separately. All analyses were performed in Stata Statistical Software version 16 [20] with weighted data unless stated otherwise. Procedures for calculating weights to be more representative of the sampled population differed between the countries [5].

\section{Results}

\section{Descriptive Information}

The characteristics of the study samples in the respective countries are presented in Table 1 . The gender distribution was similar in most countries. The mean age varied from 40.2 years in Iceland to 43.5 years in Denmark. Additionally, the estimated prevalence of non-drinkers was highest in Italy while the estimated prevalence of participants reporting weekly HED was highest in Bulgaria. The country-level proportion of monthly HED according to WHO data varied between $25.0 \%$ in Italy and $54.9 \%$ in Lithuania. Italy and Norway had the lowest level of consumption of APC, with $7.5 \mathrm{~L}$ of pure alcohol, whereas Bulgaria had the highest, with $12.7 \mathrm{~L}$.

The estimated proportions of experiencing others' alcohol-related aggression ranged from 13.2 to $46.6 \%$ among men and from 9.3 to $51.6 \%$ among women, and were lowest in Portugal for both genders and highest in Romania among men and in Lithuania among women (Fig. 1). The estimated average proportion of self-reported harm across all countries was $25 \%$ among men and 
$24 \%$ among women (online suppl. Table 1; see www.karger.com/doi/10.1159/000520079 for all online suppl. material). Being verbally abused because of someone else's drinking was the most frequently reported harm, which on average was reported by $16.5 \%$ of both men and women, followed by being in a serious argument (14.2\% men, $13.5 \%$ women) and being physically harmed (3.5\% men, $3.1 \%$ women) (online suppl. Table 1 ).

Associations between the Country-Level Prevalence of Monthly Heavy Episodic Drinking and Experiences of Others' Alcohol-Related Aggression

A $1 \%$ increase in the country-level prevalence of monthly HED was significantly associated with $5 \%$ higher odds (odds ratio [OR] 1.05) of experiencing at least 1 of 3 types of others' alcohol-related aggression among men (Table 2, HED model 1). The corresponding increased odds among women were 6\% (OR 1.06). These associations were not altered after adjustment for the country-level mean age and by including the mediator of own HED patterns (HED model 2). The intraclass correlation shows that HED at the country level accounted for $6 \%$ of the total variance of experiencing harm for both women and men.

Country-Level Prevalence of Monthly Heavy Episodic Drinking as a Modifier of the Relationship between Alcohol Consumption per Capita and Experiences of Others' Alcohol-Related Aggression

A 1-L increase in APC was significantly associated with increased odds of experiencing others' alcohol-related aggression of $14 \%$ (OR 1.14) for men and $17 \%$ (OR 1.17) for women (Table 2, APC model 1). These associations remained after adjustments for the country-level age structure and by including the mediator of own HED patterns (APC model 2). However, when including country-level HED (APC and HED model 1), the strength of the association decreased by around half to an increased odds of 9\% (OR 1.09) among men and 6\% (OR 1.06) among women. The impact of the country-wide prevalence of HED on the link between APC and experiences of others' alcohol-related aggression was further evaluated (APC and HED model 2). For both men and women, an OR of 1.01 was found, that is, the higher the country's level of HED, the stronger the association between APC and experiences of aggression related to others' drinking. However, the interaction term was only statistically significant for women and not for men.

Since the first-order variables of APC and country-level prevalence of HED were not statistically significant in

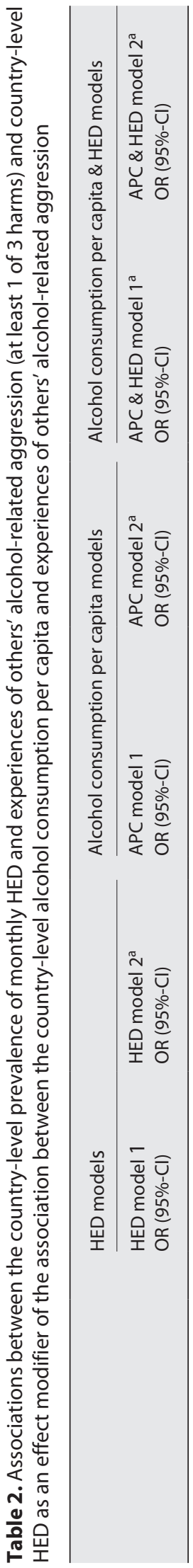

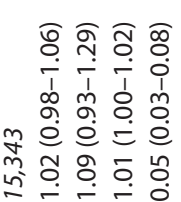

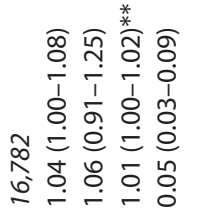




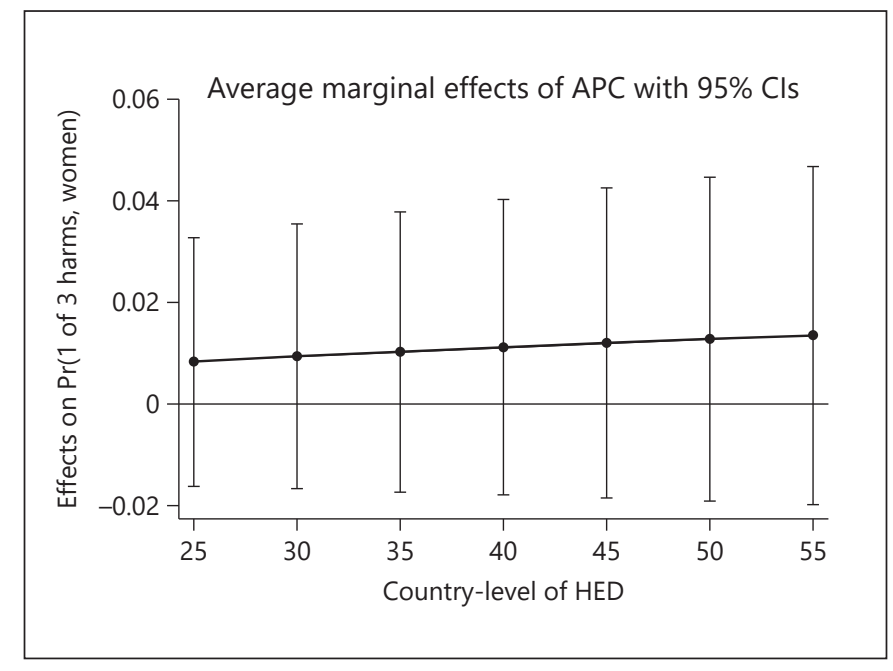

Fig. 2. Predicted probability of individual experiences of others' alcohol-related aggression (at least 1 of 3 harms) for alcohol consumption per capita (in litres of pure alcohol) at different values of country-level prevalence of monthly HED women. APC, alcohol per capita; HED, heavy episodic drinking.

the last model, the interaction term was further evaluated by assessing what a 1-unit increase in APC in litres of pure alcohol has on the probability of aggressive harm when country-level HED is held constant, for every 5 values between 25 and 55 . As the interaction term was not statistically significant for men, this evaluation was restricted to women. Figure 2 reveals an increase in the probability of aggressive harm with increasing HED, indicating that the probability of aggressive harm for APC differs at different values of HED. However, the average marginal effects are not statistically significant.

\section{Discussion}

Based on data from 19 European countries, this study has demonstrated a positive association between HED at the country level and individual-level experiences of harm from others' drinking-related aggression, that is, the higher the country-level prevalence of monthly HED, the higher the risk that an individual reports experiences of harm. This was true for both men and women. It is known that consumption of larger amounts of alcohol on a single occasion increases the risk of alcohol-related aggression $[8,9]$. Therefore, the finding that the individual exposure to others' alcohol-related aggressive behaviour was higher in countries where HED is more common was expected and in line with previous research based on data restricted at the aggregated $[10,11]$ and the individual level [14]. Nevertheless, the present findings add new knowledge to the AHTO research field by revealing that individual-level experiences of harm from others' drinking-related aggression depend not only on factors in the individual's immediate surroundings [2] but is also influenced by the general drinking pattern of the population at the societal level.

Previous studies investigating the relationship between alcohol consumption and violent and aggressive behaviour have mainly focused on the volume of drinking as an indicator at the country level [10-12]. The present study adds that the country-level HED is also important with regard to harm from others' drinking-related aggression. However, the data indicated that HED at the country level only explains a small part of the variance of this type of alcohol-related aggression (6\%). It is therefore important to study other possible factors contributing to the between-country differences in rates of these negative experiences. As suggested by researchers within the Gender and Alcohol's Harm to Others (GENAHTO) project [3], AHTO can be influenced by factors at several levels, from the individual to the regional and societal levels. Therefore, future studies should also include drinking patterns on several levels, for instance, the regional level. Factors other than drinking patterns may also play a role at the societal level. For instance, a recent study by Kilian and colleagues [21], based on the same RARHA SEAS data, revealed that the prevalence of AHTO was higher in countries with greater social inequalities than in more egalitarian countries.

The results of this study also suggested a moderating effect of country-level HED on the association between alcohol consumption per capita as an indicator of volume of drinking and drinking-related aggression: the probability of experiencing harm from others' drinking-related aggression for a 1-L increase in alcohol consumption per capita increases when the prevalence of country-level HED increases. These results are in line with findings from previous research at the aggregate level where violence has been shown to be experienced at low volumes of alcohol consumption in countries where a high proportion of drinking occasions are HED [10]. However, the moderating effect could not be established in this study because of: (a) the weak positive interaction term, (b) the non-significance of the first-order variables of alcohol consumption per capita and country-level prevalence of HED in the model including the interaction term, and (c) the non-significance of the average marginal ef- 
fects. Additionally, no statistically significant interaction was revealed among men. Hence, more evidence is needed to confirm a moderating effect of HED on the association between volume of drinking and harm from others' drinking-related aggression.

\section{Strengths and Limitations}

The major strength of this study is the comparable measurements of experiences of harm from others' alcohol-related aggression in several countries. In addition, the sample came from European countries with different drinking patterns, and outcome data were collected at approximately the same time in all countries. The different sources of information on exposure and outcome are also a major strength, as cross-sectional data are susceptible to joint misclassification if exposure and outcome are assessed through the same informants [22]. Other differences between the 2 sources may still introduce bias such as the fact that the exposure data covers a sample with a broader age range (15+ years) than the outcome data (1865 years). However, experiences of aggressive harm from others' drinking are likely to occur from exposure to heavy episodic drinkers of all ages, not only from those between 18 and 65 years. Hence, the different age ranges in the 2 samples may be less of a concern in this study.

The low response rate in some countries may be of concern in regard to the outcome measure. People who experience aggression from others could be overrepresented among the non-respondents, which may lead to different degrees of underestimation of aggressive harm across countries given that the response rate varied between the countries. However, the direction in which the non-response may bias the results is complex to predict, as low response rates occurred in countries with both low and high prevalence of the exposure measure. As an example, the non-response rate was high in Italy, a country with a low prevalence of HED, as well as in Norway, a country with a relatively high prevalence of HED. To our knowledge, non-response analyses of reports of harm from aggressions related to others' drinking have not been done. However, in a follow-up study in Sweden, participants experiencing AHTO at baseline were overrepresented among those participating in the follow-up assessment study 1 year later [23], indicating that this experience may not be a determinant of attrition. Nevertheless, it cannot be assumed that the willingness to participate in surveys on this topic is the same across countries with different drinking cultures.

The perception of and the tolerance towards alcohol consumption and alcohol-related behaviour may vary across countries with differing drinking cultures. Previous studies have suggested that survey respondents in countries with more detrimental drinking patterns may be more likely to attribute alcohol consumption to related consequences and may be more willing to report alcoholrelated problems [13]. This would suggest that experiences of harm from others' drinking-related aggression may be over-reported in countries with high prevalence of HED or underreported in countries with low prevalence of HED. Hence, the associations revealed in this study may partly reflect cross-country differences in tolerance and perception of alcohol-related harm.

\section{Conclusion}

The risk of experiencing harm from others' alcoholrelated aggression increases with increasing populationwide prevalence of HED, reinforcing the notion that these alcohol-related harms are dependent not only on factors in the individual's immediate surroundings but also on drinking patterns of the population. These findings suggest that measures lowering HED in a population might result in fewer people being exposed to aggressive behaviour related to others' drinking. However, HED at the country level explains only a small part of the variance of these harmful consequences of drinking. Thus, a more comprehensive analysis of societal factors other than HED is called for.

\section{Acknowledgement}

The authors would like to thank Thor Norström for his advice regarding the residual-centring method. The data for the Standardized European Alcohol Survey were collected within the EU Joint Action on Alcohol project co-funded by the European Commission and the Member States, in the framework of Health Programme (2008-2013).

\section{Statement of Ethics}

Informed consent to participant was not directly obtained but inferred by completion of the questionnaire/participation in the interview. Ethical approval was applied for at the Swedish Ethical Review Authority. Since the study did not include any sensitive personal information according to $\$ \$ 3-4$ Swedish Ethical Review Act, no decision was made regarding the study. The Swedish Ethical Review Authority, however, issued an advisory statement (reference number: 2020-02388) that they had no ethical objection to the study. 


\section{Conflict of Interest Statement}

The authors have no conflict of interest to declare.

\section{Funding Sources}

No external funding to declare.

\section{Author Contributions}

Erica Sundin contributed to conceptualization, drafting, design, analysis, methodology, and interpretation. Jonas Landberg contributed to conceptualization, drafting, design, methodology, and interpretation. Maria Rosaria Galanti contributed to conceptualization, design, methodology, and interpretation. Robin Room contributed to conceptualization and interpretation. Mats Ramstedt contributed to conceptualization, drafting, interpretation, project administration, and supervision.

\section{Data Availability Statement}

The data from the World Health Organization can be found in reference [15]. The RARHA SEAS data may be requested from Jacek Moskalewicz.

\section{References}

1 Room R, Laslett A-M, Jiang H. Conceptual and methodological issues in studying alcohol's harm to others. Nordisk Alkohol Nark. 2016;33(5-6):455-78.

2 Waleewong O, Laslett AM, Chenhall R, Room R. Harm from others' drinking-related aggression, violence and misconduct in five Asian countries and the implications. Int J Drug Policy. 2018 Apr 2;56:101-7.

3 Wilsnack SC, Greenfield TK, Bloomfield K. The GENAHTO project (gender and alcohol's harm to others): design and methods for a multinational study of alcohol's harm to persons other than the drinker. Int J Alcohol Drug Res. 2018;7(2):37-47.

4 Kuntsche E, Rehm J, Gmel G. Characteristics of binge drinkers in Europe. Soc Sci Med. 2004 Jul;59(1):113-27.

5 Moskalewicz J, Room R, Thom B, editors. Comparative monitoring of alcohol epidemiology across the EU. Baseline assessment and suggestions for future action. Synthesis report. Warsaw, Poland: PARPA-the State Agency for Prevention of Alcohol Related Problems; 2016.

6 Room R, Rossow I. The share of violence attributable to drinking. J Subst Use. 2001;6(8): 218-28.

7 Boles SM, Miotto K. Substance abuse and violence. Aggress Violent Behav. 2003 Mar-Apr; 8(2):155-74.

8 Graham K, Schmidt G, Gillis K. Circumstances when drinking leads to aggression: an overview of research findings. Contemp Drug Probl. 1996;23:493-557.
9 Exum ML. Alcohol and aggression: an integration of findings from experimental studies. J Crim Justice. 2006 Mar-Apr;34(2):131-45.

10 Rossow I. Alcohol and homicide: a cross-cultural comparison of the relationship in $14 \mathrm{Eu}$ ropean countries. Addiction. 2001 Feb;96 Suppl 1:S77-92.

11 Bye EK. Alcohol and homicide in Eastern Europe. Homicide Stud. 2008 Feb;12(1):7-27.

12 Ramstedt M. Population drinking and homicide in Australia: a time series analysis of the period 1950-2003. Drug Alcohol Rev. 2011 Sep;30(5):466-72.

13 Astudillo M, Kuntsche S, Graham K, Gmel G. The influence of drinking pattern, at individual and aggregate levels, on alcohol-related negative consequences. Eur Addict Res. 2010; 16(3):115-23.

14 Bye EK, Rossow I. The impact of drinking pattern on alcohol-related violence among adolescents: an international comparative analysis. Drug Alcohol Rev. 2010 Mar;29(2):131-7.

15 World Health Organization. Global status report on alcohol and health 2018. Geneva: World Health Organization; 2018. Licence: CC BY-NC-SA 3.0 IGO.

16 Rossow I, Hauge R. Who pays for the drinking? Characteristics of the extent and distribution of social harms from others' drinking. Addiction. 2004 Sep;99(9):1094-102.
17 Laslett AM, Room R, Ferris J, Wilkinson C, Livingston M, Mugavin J. Surveying the range and magnitude of alcohol's harm to others in Australia. Addiction. 2011 Sep;106(9):160311.

18 Williams R. Using the margins command to estimate and interpret adjusted predictions and marginal effects. Stata J. 2012;12(2):30831.

19 Lance CE. Residual centering, exploratory and confirmatory moderator analysis, and decomposition of effects in path models containing interactions. Appl Psychol Meas. 1988;12(2):163-75.

20 StataCorp. Stata statistical software: release 16. College Station, TX: StataCorp LLC; 2019.

21 Kilian C, Manthey J, Rehm J. Gender differences and the role of social inequality in alcohol's harm to others in Europe. J Stud Alcohol Drugs. 2020 Nov;81(6):762-9.

22 Brooks DR, Getz KD, Brennan AT, Pollack AZ, Fox PF. The impact of joint misclassification of exposures and outcomes on the results of epidemiologic research. Curr Epidemiol Rep. 2018(5):166-74.

23 Sundin E, Landberg J, Raninen J, Ramstedt M. Negativa konsekvenser av alkohol, narkotika och tobak i Sverige: en ettårsuppföljning av beroende och utsatthet för närståendes bruk [Negative consequences of alcohol, drugs and tobacco in Sweden: a one-year follow-up of dependenc and harm from others' substance use]. Stockholm: The Swedish Council for Information on Alcohol and Other Drugs; 2015. 